Relations industrielles

Industrial Relations

\title{
New Directions for Manpower Policy, par Barbara Goldman, Montréal C.D. Howe Research Institute, 1976, 113 pp.
}

Jean Sexton

Volume 33, numéro 1, 1978

URI : https://id.erudit.org/iderudit/028857ar

DOI : https://doi.org/10.7202/028857ar

Aller au sommaire du numéro

Éditeur(s)

Département des relations industrielles de l'Université Laval

ISSN

0034-379X (imprimé)

1703-8138 (numérique)

Découvrir la revue

Citer ce compte rendu

Sexton, J. (1978). Compte rendu de [New Directions for Manpower Policy, par Barbara Goldman, Montréal C.D. Howe Research Institute, 1976, 113 pp.] Relations industrielles / Industrial Relations, 33(1), 160-161.

https://doi.org/10.7202/028857ar

Tous droits réservés @ C Département des relations industrielles de l'Universite Laval, 1978
Ce document est protégé par la loi sur le droit d'auteur. L’utilisation des services d'Érudit (y compris la reproduction) est assujettie à sa politique d'utilisation que vous pouvez consulter en ligne.

https://apropos.erudit.org/fr/usagers/politique-dutilisation/ 
nous signalons ce passage: «He openly supported René Lévesque, leader of the Parti québécois, the Québec separatist party, and gave Lévesque $\$(.$.$) of his own$ money to publish a separatist oriented manifesto in 1967. His challengers claimed had channeled CNTU funds into radical political causes and that, himself had used union (...) excessively». En second lieu, il nous a semblé qu'il y aurait eu lieu de dégager d'une façon plus approfondie les dénominateurs communs et les points de différentiation des coordonnées juridiques, institutionnelles et socio-économiques des diverses provinces, quitte à atténuer l'importance démesurée accordée à l'influence américaine.

Rodrigue BLOUIN

Université Laval

New Directions for Manpower Policy, par Barbara Goldman, Montréal C. D Howe Research Institute, 1976, 113 pp.

Cet ouvrage vise essentiellement à présenter, analyser et critiquer ces instruments utilisés par le gouvernement fédéral dans le but d'améliorer le fonctionnement des marchés du travail et regroupés sous le vocable de politique de main-d'œuvre.

L'auteur, pour atteindre son objectif, présente d'abord les objectifs de la politique de main-d'œuvre canadienne et tente de résumer les principaux problèmes de marché du travail au Canada. Puis l'auteur attaque successivement la formation professionnelle comme mesure d'intervention au niveau de l'offre, la création d'emploi comme mesure d'intervention au niveau de la demande, et l'expérience des CMC comme mesure d'appariement sur les marchés du travail. Finalement, à partir de l'analyse effectuée, l'auteur présente une certaine prospective dans le domaine de la main-d'œuvre au Canada.

Il faut admettre comme jugement global, que l'ouvrage de Made Goldman est bien fait et représente un outil de travail essentiel à celui qui s'intéresse à l'aspect maind'œuvre du marché du travail. Cependant, il ne faudra pas y chercher un caractère global et général puisque plusieurs aspects de la question de la main-d'œuvre y ont été volontairement et involontairement laissés de côté. En fait, l'auteur se borne à ce qu'il a été traditionnellement conçu comme essentiel. Sur ce point, encore une fois, ce livre est intéressant. Cependant, il évite des points vitaux de la politique des services de main-d'œuvre.

D'abord, ce n'est pas cet ouvrage qui nous aidera à replacer les efforts de maind'œuvre au Canada dans une perspective historique. Cet aspect est d'autant plus important qu'il nous aidera à comprendre plusieurs des caractéristiques actuelles du système canadien de main-d'œuvre qui existent très souvent simplement parce qu'ils ont été instaurés dans des circonstances particulières par des gens identifiables pour "solutionner" des problèmes particuliers. Un tel aspect historique est d'autant plus important à examiner que le (ou les) programme (s) existe(nt) encore malgré le fait que les circonstances, les hommes et les problèmes ont profondément changé.

Ensuite, l'auteur admet (p. 12) ne se borner, à toute fin pratique, qu'aux principaux programmes qui ont traditionnellement influencé les fonctions essentielles de la politique des services de main-d'œuvre. En fait, l'auteur n'examine que l'aspect qualitatif de l'offre de travail en n'examinant que la formation professionnelle, laissant systématiquement de côté l'aspect quantitatif ( «immigration » contrôle quantitatif, et même l'aspect population à plus long terme) de cette offre de travail. Même si une telle approche est en tout point conforme à l'approche traditionnelle, un effort de «renouveau» aurait été grandement utile sur ce point.

Quant à la formation professionnelle, l'approche utilisée est à la fois conservatrice et "prudemment" novatrice. En effet, l'auteur semble se plier dans un premier temps aux objectifs traditionnels de la formation professionnelle (croissance économique, stabilisation et réduction des disparités de revenus) pour ensuite examiner d'une façon très diplomatique l'expérience d'évaluation de tels programmes qui ne se borne malheureusement qu'à des 
études coûts-bénéfices. Puis sous le titre de "Unresolved Problems», l'auteur pose des questions très pertinentes qui devront être résolues si ce programme est pour signifier quelque chose de réel dans l'avenir. Cependant, l'auteur évite soigneusement de se prononcer sur l'importance (beaucoup trop grande à mon avis) relative de la formation professionnelle dans l'effort total de main-d'œuvre et sur l'aspect institutionnel du programme qui, à date, a malheureusement vu deux juridictions intervenir, à différents degrés, dans ce domaine.

Quant à la demande de travail, l'auteur, après avoir très bien situé la nature et l'importance relative de cette fonction, présente certains programmes de création d'emplois, rend disponibles certaines données très utiles sur ces programmes et examine quelques uns des plus importants effets secondaires des efforts de création d'emploi. De façon globale, ce chapitre est. bien fait et intéressant même s'il est un peu court.

Quant à l'appariement, Made Goldman retient surtout l'expérience des CMC et l'effort de décentralisation effectué par Ottawa en matière de main-d'œuvre. Cette partie quoique intéressante, est nettement trop brève en ce qu'un effort plus minutieux aurait été nécessaire pour examiner de façon complète et critique la question délicate de l'organisation des services de main-d'œuvre au Canada.

En guise de conclusion, l'auteur s'adonne à une certaine prospective fortement inspirée des écrits et positions de l'OCDE en matière de main-d'œuvre. Cette section est d'autant plus intéressante qu'elle pique la curiosité et donne envie de poursuivre le travail.

Jean SEXTON

Université Laval

\title{
LA POLITISATION DES RELATIONS DU TRAVALL (28zme congrès 1973)
}

Introduction, Ginles LAFLAMME - Les formes historiques de politisation du syndicalisme au Québec, LÉo ROBACR - L'évolution socio-économique et le déplacement des centres de pouvoir, Bernard Solasse - L'impact des secteurs public et para-public sur la politisation des relations du travail, JEAN BoIviN - La philosophie du Code du travail, Jean-Réal Cardin - Les limites du négociable et le débordement des conflits, andré Thibaudeau - Positions des partis politiques devant la politisation des relations du travail, ROBERT Burns, André Déom, Michel Bellavance - Conséquences de la politisation des relations du travail, GERARD DION - Annexe : Négociation collective dans un monde en évolution.

1 volume, 170 pages - Prix : $\$ 5.50$

\author{
LES PRESSES DE L'UNIVERSITE LAVAL \\ CITE UNIVERSITAIRE \\ Québec, P.Q., CANADA \\ G1K 7R4
}

